\title{
INFLUÊNCIA DO DIABETES MELLITUS NO DESENVOLVIMENTO DA PERIODONTITE APICAL DE ORIGEM ENDODÔNTICA: REVISÃO DE LITERATURA
}

\section{INFLUENCE OF DIABETES MELLITUS ON THE DEVELOPMENT OF APICAL PERIODONTITIS OF ENDODONTIC ORIGIN: LITERATURE REVIEW}

Francisco Ivison Rodrigues Limeira Doutor em Clínica Odontológica, Professor do Curso de Odontologia da Faculdade Presidente Antônio Carlos, Brasil. E-mail: ivisonodontoce@hotmail.com

\section{Diandra Costa Arantes}

Doutora em Clínica Odontológica, Professora da Faculdade de Odontologia da Universidade

Federal do Pará, Brasil.

E-mail: arantesdiandra@yahoo.com.br

\section{Ana Carolina Trindade Valadares}

Especialista em Endodontia, Professora da

Faculdade São Leopoldo Mandic Belo

Horizonte, Brasil.

E-mail: carolvaladares@bol.com.br

\section{Resumo}

Este estudo teve por objetivo realizar uma revisão da literatura acerca da influência do Diabetes Mellitus (DM) no desenvolvimento da Periodontite Apical (PA) de origem endodôntica. Foi realizada uma busca na base de dados PubMed/MEDLINE. Foi utilizada uma estratégia de busca centrada nos termos relacionados ao DM e a PA, utilizando os seguintes termos MESH: "Diabetes Mellitus", "Diabetes Mellitus, Type 2", "Diabetes Mellitus, Type 1", "Hyperglycemia", "Periapical Abscess", "Periapical Granuloma", "Periapical Diseases", e "Periapical Periodontitis". Artigos publicados até junho de 2019 foram revisados. Após a busca, foi possível observar que poucos estudos investigam a associação entre o DM e a PA. Dos estudos incluídos nesta 
revisão da literatura, poucos observaram uma maior prevalência de PA na comparação entre pacientes com DM e saudáveis. Contudo, a maioria dos estudos mostra evidências de que o DM tem influência no desenvolvimento da PA e no reparo das lesões após o tratamento do canal radicular, sobretudo com influência do controle glicêmico. Foi observado que alguns estudos possuem resultados divergentes, embora mostrem algumas diferenças na história natural das lesões periapicais no paciente diabético. Assim, foi possível concluir que a maioria dos estudos indica uma influência do DM no desenvolvimento da PA, com um possível efeito adicional do controle glicêmico. No entanto, os estudos relacionados a essa temática ainda são poucos, fazendo-se necessários estudos longitudinais e ensaios clínicos bem delineados.

Palavras-chave: Diabetes Mellitus; Hiperglicemia; Periodontite Periapical.

\section{Abstract}

This study aimed to perform a literature review about the influence of Diabetes Mellitus (DM) on the development of Apical Periodontitis (AP) of endodontic origin. A search was made in the PubMed/MEDLINE database. A search strategy centered along the terms related to DM and AP (the outcome) was used. The following MESH terms were used: "Diabetes Mellitus", "Diabetes Mellitus, Type 2", "Diabetes Mellitus, Type 1", "Hyperglycemia", "Periapical Abscess", "Periapical Granuloma", "Periapical Diseases", and "Periapical Periodontitis". After the search, was observed that few studies investigate the association between DM and AP. The studies included in this literature review, few observed a higher prevalence of AP when comparing DM patients with healthy. However, most studies show evidence that DM influences the development of AP and repair of lesions after root canal treatment, especially with the influence of glycemic control. It has been observed that some studies have divergent results, although they show some differences in the natural history of periapical lesions in diabetic patients. Thus, was concluded that most studies indicate an influence of DM on AP development, with a possible additional effect of glycemic control. However, there are still few studies related to this subject, requiring longitudinal studies and welldesigned clinical trials.

Keywords: Diabetes Mellitus; Hyperglycemia; Apical Periodontitis.

\section{Introdução}

O diabetes mellitus (DM) engloba um grupo de distúrbios metabólicos altamente prevalentes que ocorrem quando o pâncreas não produz insulina suficiente (tipo 1 DMT1) ou quando o corpo não pode usar efetivamente a insulina que produz (tipo 2 DMT2). Embora o DMT2 possa ser prevenido por hábitos de vida saudáveis, ainda não existem métodos preventivos conhecidos para o DMT1 (AMERICAN DIABETES ASSOCIATION, 2017). Globalmente, estima-se que 424,9 milhões (8,8\%) de adultos (20-79 anos) viviam com DM em 2017, e esse número tem potencial para atingir 628,6 
milhões (9,9\%) em 2045 (INTERNATIONAL DIABETES FEDERATION, 2017). Em 2017, o Brasil ficou em quinto lugar entre os países com maior número de adultos com DM, com 12,5 milhões de casos, e uma estimativa de 20,3 milhões para 2045 (INTERNATIONAL DIABETES FEDERATION, 2017).

Quando não bem controlados, todos os tipos de DM podem levar a complicações em muitas partes do corpo, resultando em hospitalizações frequentes e morte precoce (INTERNATIONAL DIABETES FEDERATION, 2017). Níveis elevados persistentes de glicose no sangue causam dano vascular generalizado, resultando em dificuldades de cicatrização e em manifestações sistêmicas e orais, que afetam diretamente a integridade da polpa dentária (AHMED, 2005; LIMA et al., 2013). Existem evidências que associam o DM a uma maior prevalência de tratamento do canal radicular (CORREIA-SOUSA et al., 2015; LÓPEZ-LÓPEZ et al., 2011; SMADI, 2017), embora alguns estudos não tenham encontrado diferenças entre indivíduos com DM e não diabéticos (FALK et al., 1989; MAROTTA et al., 2012; SEGURA-EGEA et al., 2005). O prognóstico para o tratamento do canal radicular é pior em diabéticos, com maior taxa de insucesso, maior prevalência de lesões perirradiculares persistentes e extração dentária após o tratamento, e um impacto negativo na cicatrização periapical (ARYA et al., 2017; FALK et al., 1989; FOUAD e BURLESON, 2003; SEGURA-EGEA et al., 2005; WANG et al., 2013).

A periodontite apical (PA) é principalmente uma consequência da infecção do canal radicular, caracterizada por inflamação e destruição dos tecidos perirradiculares resultantes da interação entre fatores microbiológicos e resposta imune do hospedeiro (NAIR, 2004). Alguns estudos relatam uma maior prevalência de PA em indivíduos com DM (FALK et al., 1989; LÓPEZ-LÓPEZ et al., 2011; MAROTTA et al., 2012; MESGARANI et al., 2015; SÁNCHEZ-DOMÍNGUEZ et al., 2015; SEGURA-EGEA et al., 2005). Em contrapartida, outros estudos não encontraram associação significativa (BRITTO et al., 2003; CORREIA-SOUSA et al., 2015; SMADI, 2017). Estudos já mostraram uma maior frequência de lesões perirradiculares em pacientes com um longo período de diagnóstico do DM (MESGARANI et al., 2014) e associação entre o estado periapical e os níveis de hemoglobina glicada (HbA1c) (SÁNCHEZDOMÍNGUEZ et al., 2015).

A literatura científica mostra evidências confiáveis de uma associação entre o 
DM e a doença periodontal (BORGNAKKE et al., 2013; WANG et al., 2014). No entanto, a relação entre o DM e a PA de origem endodôntica permanece não comprovada, pois os estudos disponíveis parecem controversos.

O objetivo deste estudo foi realizar uma revisão da literatura acerca da influência do DM no desenvolvimento da PA de origem endodôntica.

\section{Revisão de literatura}

Foi realizada uma busca na base de dados PubMed/MEDLINE (https://www.ncbi.nlm.nih.gov/pubmed/). Foi utilizada uma estratégia de busca centrada nos termos relacionados ao DM e PA, utilizando os seguintes termos MESH: "Diabetes Mellitus", "Diabetes Mellitus, Type 2", "Diabetes Mellitus, Type 1", "Hyperglycemia”, "Periapical Abscess", "Periapical Granuloma”, "Periapical Diseases", e "Periapical Periodontitis".

Estudos em humanos avaliando a influência do DM no desenvolvimento da PA ou na cicatrização da PA após o tratamento do canal radicular foram incluídos nesta revisão. Não foram utilizados filtros que pudessem restringir o período de publicação do artigo, e apenas artigos publicados em inglês foram incluídos. Todos os artigos publicados até junho de 2019 foram revisados e alguns estudos relevantes encontrados nas listas de referência dos artigos selecionados também foram incluídos. Publicações que não atendiam a esses critérios de inclusão, como estudos em animais, relatos de casos, séries de casos e revisões de literatura foram excluídas.

A primeira associação entre o DM e a PA foi reportada em um estudo na década de 1960, por Cheraskin e Ringsdorf (1968). Os níveis de glicose de 25 pacientes foram analisados e os mesmos divididos em dois grupos: nível de glicose recomendado (12 pacientes) e nível de glicose acima do recomendado (13 pacientes). Todos os pacientes apresentavam um dente com PA e os mesmos receberam tratamento endodôntico não cirúrgico convencional. Após 30 semanas de monitoramento por análise radiográfica periapical, foi observado que o grupo com níveis de glicose dentro do recomendado mostrou uma redução de $74 \%$ no tamanho da PA, enquanto o outro grupo teve apenas uma redução de $48 \%$. 
Falk et al. (1989) avaliaram a prevalência de PA em pacientes com DMT1 de longa duração (28,9 \pm 10,19 anos) e de curta duração (5,2 \pm 1,92 anos), e compararam com pacientes saudáveis por meio de análises clínicas e radiográficas. Não houve diferença significativa na prevalência de PA em dentes tratados endodonticamente entre pacientes diabéticos e não diabéticos. No entanto, os resultados mostraram que mulheres com DMT1 de longa duração tinham mais dentes que necessitavam de tratamento do canal radicular e maior prevalência de PA do que os outros grupos (mulheres com DMT1 de curta duração ou mulheres sem DMT1).

Em um estudo retrospectivo, Fouad e Burleson (2003) avaliaram os prontuários clínicos de pacientes submetidos ao tratamento do canal radicular. Um total de 5.494 casos, incluindo 284 casos em pacientes com DM, foram tratados e 540 casos, incluindo 73 casos em pacientes com DM, tiveram os dados de acompanhamento de dois anos ou mais no pós-operatório analisados. Após a coleta de dados, a análise estatística revelou que a taxa de sucesso foi significativamente menor em pacientes diabéticos com lesões pré-operatórias do que em indivíduos sem DM. Outro resultado importante foi encontrado através da análise multivariada, que mostrou que pacientes sem DM tiveram uma maior chance de sucesso no tratamento endodôntico de dentes com lesões periapicais pré-operatórias do que pacientes com DM (odds ratio $=8,13$ ). Assim, os autores concluíram que pacientes com DM tratados endodonticamente devem ser avaliados cuidadosamente e tratados com regimes antimicrobianos eficazes no canal radicular, principalmente nos casos com lesões pré-operatórias.

Britto et al. (2003) investigaram a prevalência de PA em dentes tratados endodonticamente e não tratados em 30 pacientes com DM e 23 sem DM. Não foram observados efeitos principais do sexo, diagnóstico do DM ou idade (covariável) nos desfechos de interesse (dentes sem tratamento endodôntico com PA, dentes tratados endodonticamente com PA e dentes tratados endodonticamente sem PA). No entanto, houve interações significativas entre o diagnóstico de PA, sexo e DM para ambos os resultados endodônticos, dentes tratados endodonticamente com PA e dentes tratados endodonticamente sem PA. Isso significou que homens com DMT2 que tinham tratamentos endodônticos estavam mais propensos ao surgimento de PA após o tratamento. Com isto, os autores concluíram que o DMT2 está associado a um risco 
aumentado de resposta negativa dos tecidos perirradiculares à patógenos odontogênicos.

Segura-Egea et al. (2005) realizaram um estudo transversal para avaliar a prevalência de PA em pacientes com DMT2 e indivíduos controles sem DM, por meio da análise de radiografias periapicais. A região periapical de todos os dentes, excluindo terceiros molares, foi examinada e o estado periapical foi avaliado pelos escores do Índice Periapical. Quando o sujeito foi considerado como unidade de análise, a prevalência de PA em pacientes diabéticos foi de $81 \%$ e no grupo controle foi de 58\%. Assim, pacientes com DM tiveram chance três vezes maior de apresentar lesões periapicais do que pacientes saudáveis (odds ratio = 3,2). Quando os dentes tratados endodonticamente foram considerados, não houve diferença estatisticamente significativa na porcentagem de lesões periapicais entre pacientes com ou sem DM ( $p$ $>0,05)$. No entanto, quando consideraram apenas dentes sem tratamento do canal radicular, pacientes com DM tiveram o dobro de dentes com PA (odds ratio =2.1). Os autores sugerem a existência de algumas diferenças na história natural das lesões periapicais no paciente diabético e apoiam fortemente o conceito de que o DM atua como fator de risco para a PA, aumentando sua prevalência e podendo afetar o resultado do tratamento do canal radicular.

López-López et al. (2011) investigaram radiograficamente a prevalência de PA e tratamento endodôntico em uma amostra de 50 pacientes adultos com DMT2, bem controlados, e de 50 indivíduos sem DM. A condição periapical foi avaliada pelos escores do Índice Periapical. PA em um ou mais dentes foi encontrada em $74 \%$ dos pacientes diabéticos e em $42 \%$ dos indivíduos controle (odds ratio $=3,9$ ). Entre os pacientes diabéticos com dentes tratados endodonticamente, 46\% apresentaram PA afetando pelo menos um dente tratado. Entre os pacientes controles com dentes tratados endodonticamente, 24\% tiveram PA afetando pelo menos um dente ( $p>$ 0,05). A associação entre o DM e a maior prevalência de PA permaneceu inalterada após análise multivariada (odds ratio $=3,3$ ). Assim, os resultados mostraram que em pacientes adultos o DMT2 está significativamente associado ao aumento da prevalência de PA e tratamento endodôntico.

Wang et al. (2011) analisaram o impacto de doenças sistêmicas (DM, hipertensão arterial e doença arterial coronariana) no risco de extração dentária após o 
tratamento não cirúrgico do canal radicular. Um total de 49.334 dentes foi acompanhado por dois anos. Dos dentes analisados, 3,2\% foram extraídos durante o período de dois anos de seguimento. Foi observado que todas as doenças sistêmicas avaliadas são fatores de risco significativos para a extração dentária após o tratamento endodôntico (DM - hazard ratio $=1,79)$, sobretudo nos casos com lesões perirradiculares pré-operatórias.

Marotta et al. (2012) avaliaram a prevalência de PA e tratamento endodôntico em 30 indivíduos com DMT2 em comparação com 60 não diabéticos de uma população adulta brasileira, pareados por sexo e idade, através da análise de radiografias periapicais digitais. Em relação aos indivíduos, não houve diferença significativa na prevalência de PA entre os grupos com ou sem DMT2. No entanto, quando os dentes foram a unidade de análise, os indivíduos com DMT2 apresentaram uma maior quantidade de dentes com PA do que os indivíduos do grupo controle. Além disso, os autores mostraram que a prevalência de PA em dentes sem tratamento endodôntico foi significativamente maior no grupo com DMT2, no entanto, em dentes com tratamento endodôntico, essas diferenças não foram percebidas. Diante dos resultados encontrados, os autores concluíram que a PA foi significativamente mais prevalente em dentes não tratados de pacientes com DMT2. Além do mais, sugeriram que o DMT2 pode servir como um modificador da lesão periapical, no sentido de que indivíduos com DMT2 podem ser mais propensos a desenvolver a doença primária. No entanto, os achados não confirmam que o DM possa influenciar a resposta ao tratamento de canal radicular, porque os dentes tratados não apresentaram maior prevalência de PA quando comparados aos controles.

Mesgarani et al. (2014) avaliaram a frequência de lesões perirradiculares em pacientes com DM. Os pacientes foram divididos em grupos de acordo com o tempo de diagnóstico do DM, sendo de longo prazo, quando acima de 48 meses, e de curto prazo, quando abaixo de 48 meses. Os pacientes também foram divididos segundo o controle do DM, bem controlado $(\mathrm{HbA} 1 \mathrm{c}<7)$ e mal controlado $(\mathrm{HbA} 1 \mathrm{c} \geq 7)$. Foram observadas lesões perirradiculares em $90,37 \%$ dos pacientes. A frequência de PA em pacientes com longo prazo de diagnóstico do DM foi de $94,4 \%$ e de $82,2 \%$ naqueles com curto prazo $(p=0,023)$. Não foram observadas diferenças na prevalência de PA 
entre os pacientes quanto ao controle do DM. Assim, os autores reforçam que o tempo de diagnóstico do DM pode influenciar no surgimento de lesões perirradiculares.

Correia-Sousa et al. (2015) investigaram a possível associação entre a prevalência de PA e algumas condições sistémicas (doenças cardiovasculares, DM, alergias e tabagismo) numa população adulta. Registros dos tratamentos endodônticos de 421 pacientes (10.540 dentes) foram analisados. O status periapical foi avaliado através de radiografias panorâmicas e classificado de acordo com o Índice Periapical. Foi observada uma maior prevalência de dentes com tratamento endodôntico em indivíduos com DM, entretanto, não foi observada uma associação significativa entre a prevalência de PA e o DM. Assim, os autores sugerem que a influência de fatores de risco, como o DM, relacionados com o prognóstico do tratamento endodôntico e da PA é uma ferramenta válida para a decisão de tratamento do clínico.

Sánchez-Domínguez et al. (2015) analisaram a existência de associação entre a prevalência de PA e o controle glicêmico de pacientes com DMT2, por meio dos níveis de HbA1c. A amostra de 83 pacientes foi dividida em dois grupos de acordo com o controle do DM, grupo com bom controle ( $n=24, \mathrm{HbA} 1 \mathrm{c}<6,5 \%)$ e o grupo de pobre controle $(n=59, \mathrm{HbA} 1 \mathrm{c} \geq 6,5 \%)$. Não houve diferença significativa entre os grupos quando avaliada a prevalência de indivíduos com PA em dentes com e sem tratamento do canal. A análise de regressão logística multivariada mostrou que o pior estado periapical se correlacionou significativamente com os níveis de $\mathrm{HbA} 1 \mathrm{c} \geq 6,5 \%$ em pacientes com DMT2 (odds ratio $=3,8$ ). Assim, os autores concluíram que os níveis de $\mathrm{HbA} 1 \mathrm{c}$ em pacientes diabéticos estão associados ao estado periapical. Além do que, os resultados encontrados sustentam ainda mais a relação entre o controle glicêmico do DM e a inflamação periapical.

Arya et al. (2017) compararam o sucesso do tratamento do canal radicular primário entre pacientes com DMT2 e não diabéticos, investigando o efeito dos níveis de $\mathrm{HbA1c}$ na cura periapical dos dentes de pacientes diabéticos com PA. Sessenta molares inferiores com polpa necrótica e PA foram incluídos no estudo. Com base nos níveis de $\mathrm{HbA1c}$, os pacientes foram divididos em 2 grupos: diabéticos (HbA1c $\geq$ $6,5 \%)$ e não diabéticos ( $\mathrm{HbA} 1 \mathrm{c}<6,5 \%)$. Quarenta e seis dentes foram avaliados no período de 12 meses e o desfecho foi analisado conforme o Índice Periapical. Foi 
observada nos dois grupos uma redução significativa no escore periapical após tratamento endodôntico nos meses de acompanhamento. Uma cura periapical significativamente menor foi observada no grupo diabético (43\%) em comparação com o grupo não diabético (80\%). Assim, os autores concluíram que o DMT2 pode ter um impacto negativo no resultado do tratamento endodôntico em termos de cicatrização periapical.

Smadi (2017) investigou a prevalência de PA em pacientes com DM em comparação com pacientes não diabéticos e avaliou o efeito do controle glicêmico na prevalência de PA. Os pacientes com DM foram divididos de acordo com o nível de controle glicêmico em dois subgrupos, DM bem controlado e DM mal controlado. Os escores do Índice Periapical foram utilizados para avaliar o status periapical. A prevalência de PA foi maior nos pacientes com DM do que no grupo com os não diabéticos, 13,5 e 11,9\%, respectivamente. O grupo com DM mal controlado teve uma maior prevalência de PA em comparação com o grupo com DM bem controlado, $18,29 \%$ e 9,21\%, respectivamente. Diante dos achados, o autor concluiu que um controle glicêmico inadequado pode estar associado ao aumento da prevalência de PA. Assim, o aconselhamento de pacientes diabéticos, particularmente aqueles com controle glicêmico inadequado, sobre o risco de falha do tratamento endodôntico deve fazer parte do protocolo clínico do profissional.

\section{Discussão}

A doença periodontal e a PA de origem endodôntica compartilham um conteúdo bacteriano semelhante, que compreende principalmente bactérias anaeróbias gramnegativas (GOMES et al., 2004; NOIRI e EBISU, 2001). Além disso, altos níveis de mediadores pró-inflamatórios estão presentes em ambas as condições (BARKHORDAR et al., 1999; GAMONAL et al., 1999). A literatura apresenta várias linhas de evidência mostrando uma correlação entre o DM e a doença periodontal (GENCO, 1996; KARJALAINEN et al., 1994; MEALEY e OATES, 2006). Apesar de haver semelhanças entre essas patologias, os resultados dos estudos referentes à associação entre o DM e a PA parecem inconclusivos, pois há poucos relatos sobre o assunto. 
Dos estudos incluídos nesta revisão da literatura, somente os estudos de Segura-Egea et al. (2005), López-López et al. (2011) e Smadi (2017) encontraram uma maior prevalência de PA em pacientes com DM em comparação com indivíduos sem DM. Enquanto os estudos de Falk et al. (1989), Britto et al. (2003), Marotta et al. (2012) não mostraram diferenças na prevalência de PA na comparação entre grupos de pacientes com DM e sem DM, e o estudo de Correia-Sousa et al. (2015) não encontraram associação entre o DM e a PA. Embora estes estudos não tenham encontrado diferença e associação significativas na prevalência de PA em pacientes com DM, os mesmos apresentam outros resultados que podem dar indícios da influência do DM no desenvolvimento da PA. Falk et al. (1989) encontraram uma maior prevalência de PA em mulheres com DMT1. Britto et al. (2013) mostraram que homens com DMT2 tem uma maior chance de insucesso no tratamento do canal devido a persistência da lesão periapical. Marotta et al. (2012) encontraram uma maior quantidade de dentes com PA em pacientes com DM, bem como, uma maior quantidade de dentes com tratamento do canal radicular apresentando PA. Além do mais, Arya et al. (2017), Fouad e Burleson (2003) e Wang et al. (2011) mostraram que pacientes com DM possuem um risco aumentado de insucesso no tratamento do canal radicular, principalmente em casos com PA pré-operatória.

A possível associação entre o DM e a PA pode ser justificada devido ao comprometimento da função das células imunes. As citocinas pró-inflamatórias, monócitos e leucócitos polimorfonucleares são regulados em excesso e os fatores de crescimento e macrófagos, são regulados negativamente, predispondo a inflamação crônica, ruptura progressiva dos tecidos e diminuição na capacidade de reparação tecidual (IACOPINO, 2001). Além disso, pacientes diabéticos apresentam níveis aumentados de produtos finais da glicação avançada (AGEs), que interagem com os receptores da superfície celular, aumentando o estresse oxidativo nos tecidos e regulando positivamente a resposta inflamatória (FOUAD e HUANG, 2015). Em diabéticos mal controlados, a resposta imune é ainda mais diminuída com a função leucocitária diminuída e retardo na cicatrização (FOUAD e HUANG, 2015; IACOPINO, 2001).

Com exceção do estudo de Falk et al. (1989), os demais estudos incluídos nesta revisão analisaram pacientes com DMT2 ou amostras combinadas de DMT1 e 
DMT2. O DM tem efeito direto na integridade da polpa dentária, e o controle glicêmico deficiente do DM pode ter um impacto negativo nessa relação (LIMA et al., 2013). As duas principais formas de DM - DMT1 e DMT2 - são caracterizadas pela insuficiência progressiva das células $\beta$ e hiperglicemia. No DMT1, isso geralmente é causado por um ataque autoimune contra as células $\beta$, induzindo a morte progressiva das células. No momento do diagnóstico, a massa de células $\beta$ é reduzida em 70 a $80 \%$. A patogênese do DMT2 é mais variável, compreendendo diferentes graus de falha das células $\beta$ em relação a diferentes graus de resistência à insulina, com perda de células $\beta$ de 25 a 50\% (CNOP et al., 2005). O DMT1 tem um efeito mais grave nos tecidos (American Diabetes Association, 2014). Assim, a comparação entre estudos com diferentes tipos de DM deve ser feita com reserva, pois podem existir diferenças na influência dos diferentes tipos de DM no desenvolvimento da PA.

Cheraskin e Ringsdor (1968) e Smadi et al. (2017) mostraram que o controle glicêmico tem efeito na prevalência de PA e no reparo das lesões após o tratamento do canal radicular. Em contrapartida, Mesgarani et al. (2014) e Sánchez-Domínguez et al. (2015) não encontraram diferenças quanto a prevalência de PA entre pacientes com e sem o controle do DM. Todavia, Sánchez-Domínguez et al. (2015) mostraram que um pior estado periapical (presença de PA) está correlacionado com os níveis de $\mathrm{HbA} 1 \mathrm{c}$ acima do recomendado em pacientes com DMT2.

Acredita-se que a hiperglicemia prejudica a circulação colateral e pode causar diversas alterações nas estruturas pulpar e periapical (BENDER e BENDER, 2003, LIMA et al., 2013). Níveis altos de glicose podem inibir a função dos macrófagos, resultando em um estado inflamatório que prejudica a proliferação celular do hospedeiro e atrasa a cicatrização da polpa e dos tecidos periapicais (GARBER et al., 2009). Por outro lado, a ação de mediadores inflamatórios liberados na inflamação periapical está associada ao desenvolvimento de resistência à insulina (PICKUP, 2004).

Segura-Egea et al., (2016) realizaram uma revisão sistemática e metanálise investigando a possível associação entre o DM e a falha do tratamento do canal radicular, avaliada como a prevalência de lesões periapicais em dentes tratados endodonticamente. Os autores concluíram que as evidências científicas disponíveis indicam que o DM está significativamente associado à maior prevalência de 
radiolucências periapicais em dentes tratados endodonticamente. Os autores ainda reforçam que levando em conta que o DM é a terceira condição médica crônica mais prevalente entre os pacientes odontológicos, os profissionais devem estar cientes da relação entre o resultado do tratamento endodôntico e o DM.

Os resultados dos estudos realizados até o momento, não são conclusivos, mas mostram algumas diferenças na história natural das lesões periapicais no paciente diabético, sugerindo uma associação entre o DM e a PA. O DM e a PA estão associados a fatores de risco comuns, mas sem a existência de uma relação de causa-efeito entre eles (SEGURA-EGEA et al., 2019). Assim, são necessários estudos epidemiológicos prospectivos para aprofundar o entendimento da relação entre a doença inflamatória de origem endodôntica e o DM.

\section{Considerações finais}

Considerando os estudos incluídos nesta revisão da literatura, a maioria indica uma influência do DM no desenvolvimento da PA de origem endodôntica, com um possível efeito adicional do controle glicêmico. No entanto, os estudos relacionados a essa temática ainda são poucos, fazendo-se necessários estudos longitudinais e ensaios clínicos bem delineados, que possam fornecer evidências científicas mais robustas.

\section{Referências}

AHMED, N. Advanced glycation end products: role in pathology of diabetic complications. Diabetes Res Clin Pract, v. 67, n. 1, p. 3-21, 2005.

AMERICAN DIABETES ASSOCIATION. Diagnosis and Classification of Diabetes Mellitus. Diabetes Care, v. 37, suppl. 1, p. S81-S90, 2014.

AMERICAN DIABETES ASSOCIATION. Standards of Medical Care in Diabetes 2017. Diabetes Care, v. 40, n. 1, p. S1-S135, 2017. 
ARYA, S.; DUHAN, J.; TEWARI, S.; SANGWAN, P.; GHALAUT, V.; AGGARWAL, S. Healing of Apical Periodontitis after Nonsurgical Treatment in Patients with Type 2 Diabetes. J Endod, v. 43, n. 10, p. 1623-7, 2017.

BARKHORDAR, R. A.; HAYASHI, C.; HUSSAIN, M. Z. Detection of interleukin-6 in human dental pulp and periapical lesions. Endod Dent Traumatol, v. 15, n. 1, p. 26-7, 1999.

BENDER, I. B.; BENDER, A. B. Diabetes mellitus and the dental pulp. J Endod, v. 29, n. 6, p. 383-9, 2003.

BORGNAKKE, W. S.; YL€OSTALO, P. V.; TAYLOR, G. W.; GENCO, R. J. Effect of periodontal disease on diabetes: systematic review of epidemiologic observational evidence. J Periodontol, v. 84, p. S135-52, 2013.

BRITTO, L. R.; KATZ, J.; GUELMANN, M.; HEFT, M. Periradicular radiographic assessment in diabetic and control individuals. Oral Surg Oral Med Oral Pathol Oral Radiol Endod, v. 96, n. 4, p. 449-52, 2003.

CHERASKIN, E.; RINGSDORF, W. M. The biology of the endodontic patient. 3. Variability in periapical healing and blood glucose. J Oral Med, v. 23, n. 3, p. 87-90, 1968.

CNOP, M.; WELSH, N.; JONAS, J. C.; JÖRNS, A.; LENZEN, S.; EIZIRIK, D. L. Mechanisms of pancreatic beta cell death in type 1 and type 2 diabetes: many differences, few similarities. Diabetes, v. 54, suppl. 2, p. S97-107, 2005. 
CORREIA-SOUSA, J.; MADUREIRA, A. R.; CARVALHO, M. F.; TELES, A. M.; PINAVAZ, I. Apical periodontitis and related risk factors: cross-sectional study. Rev Port Estomatol Cir Maxilofac, v. 56, n. 4, p. 226-32, 2015.

FALK, H.; HUGOSON, A.; THORSTENSSON, H. Number of teeth, prevalence of caries and periapical lesions in insulin-dependent diabetics. Scand J Dent Res, v. 97, n. 3, p. 198-206, 1989.

FOUAD, A. F.; BURLESON, J. The effect of diabetes mellitus on endodontic treatment outcome: data from an electronic patient record. J Am Dent Assoc, v. 134, n. 1, p. 4351, 2003.

FOUAD, A. F.; HUANG, G. T-J. Chapter 9: inflammation and Immunological response. In: Rotstein I. Ingle's Endodontics. 7th Ed. Eurospan, 2015.

GAMONAL, J.; ACEVEDO, A.; BASCONES, A.; JORGE, O.; SILVA, A. Levels of interleukin-1 beta, -8 , and -10 and RANTES in gingival crevicular fluid and cell populations in adult periodontitis patients and the effect of periodontal treatment. $\mathbf{J}$ Periodontol, v. 71, n. 10, p. 1535-45, 2000.

GARBER, S. E.; SHABAHANG, S. ESCHER, A. P.; TORABINEJAD, M. The effect of hyperglycemia on pulpal healing in rats. J Endod, v. 35, n. 1, p. 60-2, 2009.

GENCO, R. J. Current view of risk factors for periodontal diseases. J Periodontol, v. 67, suppl 10, p. 1041-9, 1996.

GOMES, B. P.; PINHEIRO, E. T.; GADÊ-NETO, C. R.; SOUSA, E. L.; FERRAZ, C. C.; ZAIA, A. A.; TEIXEIRA, F. B.; SOUZA-FILHO, F. J. Microbiological examination of infected dental root canals. Oral Microbiol Immunol, v. 19, n. 2, p. 71-6, 2004. 
IACOPINO, A. M. Periodontitis and diabetes interrelationships: role of inflammation. Ann Periodontol, v. 6, n. 1, p. 125-37, 2001.

INTERNATIONAL DIABETES FEDERATION. IDF Diabetes Atlas. 8th ed. Brussels, Belgium: International Diabetes Federation, 2017.

KARJALAINEN, K. M.; KNUUTTILA, M. L.; VON DICKHOFF, K. J. Association of the severity of periodontal disease with organ complications in type 1 diabetic patients. $\mathbf{J}$ Periodontol, v. 65, n. 11, p. 1067-72, 1994.

LIMA, S. M.; GRISI, D. C.; KOGAWA, E. M.; FRANCO, O. L.; PEIXOTO, V. C.; GONÇALVES-JÚNIOR, J. F.; ARRUDA, M. P.; REZENDE, T. M. Diabetes mellitus and inflammatory pulpal and periapical disease: a review. Int Endod J, v. 46, n. 8, p. 700-9, 2013.

LÓPEZ-LÓPEZ, J.; JANÉ-SALAS, E.; ESTRUGO-DEVESA, A.; VELASCO-ORTEGA, E.; MARTÍN-GONZÁLEZ, J.; SEGURA-EGEA, J. J. Periapical and endodontic status of type 2 diabetic patients in Catalonia, Spain: a cross-sectional study. J Endod, v. 37, n. 5, p. 598-601, 2011.

MAROTTA, P. S.; FONTES, T. V.; ARMADA, L.; LIMA, K. C.; RÔÇAS, I. N.; SIQUEIRA, J. F. JR. Type 2 diabetes mellitus and the prevalence of apical periodontitis and endodontic treatment in an adult Brazilian population. J Endod, v. 38, n. 3, p. 297$300,2012$.

MEALEY, B. L.; OATES, T. W. Diabetes mellitus and periodontal diseases. $\mathbf{J}$ Periodontol, v. 77, n. 8, p. 1289-303, 2006. 
MESGARANI, A.; HAGHANIFAR, S.; ESHKEVARI, N.; EHSANI, M.; KHAFRI, S.; NAFARZADE, S.; DAMANKESH, Z. Frequency of odontogenic periradicular lesions in diabetic patients. Caspian J Intern Med, v. 5, n. 1, p. 22-5, 2014.

NAIR, P. N. Pathogenesis of apical periodontitis and the causes of endodontic failures. Crit Rev Oral Biol Med, v. 15, n. 6, p. 348-81, 2004.

NOIRI, Y.; LI, L.; EBISU, S. The localization of periodontal-disease-associated bacteria in human periodontal pockets. J Dent Res, v. 80, n. 10, p. 1930-4, 2001.

PICKUP, J. C. Inflammation and activated innate immunity in the pathogenesis of type 2 diabetes. Diabetes Care, v. 27, n. 3, p. 813-23, 2004.

SÁNCHEZ-DOMÍNGUEZ, B.; LÓPEZ-LÓPEZ, J.; JANÉ-SALAS, E.; CASTELLANOSCOSANO, L.; VELASCO-ORTEGA, E.; SEGURA-EGEA, J. J. Glycated hemoglobin levels and prevalence of apical periodontitis in type 2 diabetic patients. J Endod, v. 41, n. 5, p. 601-6, 2015.

SEGURA-EGEA, J. J.; CABANILLAS-BALSERA, D.; JIMÉNEZ-SÁNCHEZ, M. C.; MARTÍN-GONZÁLEZ, J. Endodontics and diabetes: association versus causation. Int Endod J, v. 52, n. 6, p. 790-802, 2019.

SEGURA-EGEA, J. J.; JIMENEZ-PINZON, A.; RIOS-SANTOS, J. V.; VELASCOORTEGA, E.; CISNEROS-CABELLO, R.; POYATO-FERRERA, M. High prevalence of apical periodontitis amongst type 2 diabetic patients. Int Endod J, v. 38, n. 8, p. 564-9, 2005.

SEGURA-EGEA， J. J.; MARTÍN-GONZÁLEZ, J.; CABANILLAS-BALSERA， D.; FOUAD, A. F.; VELASCO-ORTEGA, E.; LÓPEZ-LÓPEZ, J. Association between 
diabetes and the prevalence of radiolucent periapical lesions in root-filled teeth: systematic review and meta-analysis. Clin Oral Investig, v. 20, n. 6, p. 1133-41, 2016.

SMADI, L. Apical periodontitis and endodontic treatment in patients with type II diabetes mellitus: comparative cross-sectional survey. J Contemp Dent Pract, v. 18, n. 5, p. 358-62, 2017.

SALOMAO, P. E. A. et al. As Tecnologias de Informação e Comunicação (TIC) no ensino superior. Revista Multidisciplinar do Nordeste Mineiro, v. 1, 2018.

WANG, C. H.; CHUEH, L. H.; CHEN, S. C.; FENG, Y. C.; HSIAO, C. K.; CHIANG, C. P. Impact of diabetes mellitus, hypertension, and coronary artery disease on tooth extraction after nonsurgical endodontic treatment. J Endod, v. 37, n. 1, p. 1-5, 2011.

WANG, T. F.; JEN, I. A.; CHOU, C.; LEI, Y. P. Effects of periodontal therapy on metabolic control in patients with type 2 diabetes mellitus and periodontal disease: a meta-analysis. Medicine (Baltimore), v. 93, n. 28, e292, 2014. 